\title{
ELSA cohorte 2014: clases de consumo de alcohol, tabaco y marihuana en estudiantes universitarios argentinos
}

\author{
ELSA 2014 Cohort: Classes of filcohol, Tobacco and IIarijuana Use fimong firgentinian \\ College Students

\section{Cohort elSf 2014: classes de consumo de álcool, tabaco e maconha em estudantes universitários argentinos}

\author{
Belén del Valle Vera ${ }^{*}{ }^{* *}$ \\ Angelina Pilatti*,** \\ Ricardo Marcos Pautassi ${ }^{*}{ }^{* * *}$ \\ Universidad Nacional de Córdoba, Facultad de Psicología* \\ Instituto de Investigaciones Psicológicas, IIPSI, UNC-CONICET ${ }^{* *}$ \\ Instituto de Investigación Médica Mercedes y Martín Ferreyra, INIMEC-CONICET-UNC ${ }^{* * *}$
}

Doi: https://doi.org/10.12804/revistas.urosario.edu.co/apl/a.9445

\section{Resumen}

El consumo de sustancias es altamente prevalente en estudiantes universitarios de Argentina y el mundo. Diferenciar consumidores con diversos patrones de consumo de sustancias es importante para identificar aquellos más vulnerables a avanzar hacia un consumo patológico. Por ello, el objetivo fue identificar, mediante Análisis de Clases Latentes (ACL) aplicado a diferentes indicadores de consumo de alcohol, tabaco y marihuana, las clases de consumidores y observar si dichas clases diferían en la densidad de familiares con abuso de alcohol y otros indicadores de consumo. El análisis se realizó en un total de 4.497 estudiantes de primer año que completaron instrumentos para medir: 1-consumo de alcohol, 2-consecuencias negativas del consumo de alcohol y 3-historia familiar de abuso de alcohol. Así se identificaron cuatro clases de consumidores: 1-CATM: Consumo Elevado de Alcohol, Consumo de Tabaco y Marihuana; 2-CMEA: Consumo Moderado/Elevado de Alcohol, Sin Consumo de Otras Sustancias; 3-CMBA:

Belén del Valle Vera ORCID ID: https://orcid.org/0000-0002-7840-9493

Angelina Pilatti ORCID ID: https://orcid.org/0000-0002-7277-0835

Ricardo Marcos Pautassi ORCID ID: https://orcid.org/0000-0003-1876-2321

Dirigir la correspondencia a Belén del Valle Vera. Dirección: Enfermera Gordillo esquina Enrique Barros s/n, 3er piso, Córdoba, Argentina. Teléfono: 54-351-5353890,interno: 60201. Correo electrónico: belen.vera@unc.edu.ar

Este trabajo ha sido posible gracias al financiamiento de la Agencia Nacional de Promoción Científica y Tecnológica (Foncyt) y de la Secretaría de Ciencia y Tecnología de la Universidad Nacional de Córdoba (SECyT-UNC) a nombre de la Dra. Angelina Pilatti. Quienes realizamos este trabajo queremos agradecer a quienes participaron de este estudio completando la encuesta, y a todos/as los/as estudiantes avanzados/as de psicología que colaboraron en la recolección de datos.

Conflicto de intereses: los autores declaramos no tener conflictos de intereses que declarar.

Para citar este artículo: Vera, B. del V., Pilatti, A., \& Pautassi, R. M. (2021). ELSA cohorte 2014: clases de consumo de alcohol, tabaco y marihuana en estudiantes universitarios argentinos. Avances en Psicología Latinoamericana, 39(2), 1-19. https://doi.org/10.12804/ revistas.urosario.edu.co/apl/a.9445 
Consumo Moderado/Bajo de Alcohol, Sin Consumo de Otras Sustancias; y 4-SC: Sin Consumo de Sustancias. Los miembros de las clases CATM y CMEA reportaron niveles de consumo de alcohol significativamente más elevados que el resto de las clases. Los integrantes de CATM presentaron consumo extremo de alcohol. Las clases SC y CATM exhibieron mayor densidad de familiares con abuso de alcohol que las otras clases. La mayor parte de los encuestados fueron clasificados en categorías de consumo de alto riesgo, caracterizadas por co-uso de sustancias o por consumo elevado de alcohol. Estos patrones de consumo se han asociado a numerosas consecuencias negativas.

Palabras clave: alcohol; tabaco; marihuana; clases de consumo; estudiantes universitarios.

\section{fbstract}

Substance use is highly prevalent among college students in Argentina and the world. Differentiating users with different patterns of substance use is important to identify those at a higher vulnerability to progress towards pathological use. The aims were to identify, by means of Latent Class Analysis applied to different alcohol, tobacco, and marijuana use indicators, the classes of substance use and analyze whether these classes exhibit differences in the density of relatives with a history of alcohol abuse and other alcohol use indicators. A total of 4.497 freshmen completed instruments to measure: 1- alcohol, tobacco, and marijuana use, 2- alcohol related negative consequences, and 3- family history of alcohol abuse. Four classes of substance users were identified: 1- HATM: heavy alcohol use, with tobacco and marijuana use, 2- $M H A$ : moderate/high alcohol use, without other substances, 3-MLA: moderate/low alcohol use, without other substances, and 4- NS: no substance use. The members of the HATM and MHA classes reported significantly higher levels of alcohol use than the rest of the classes. HATM members presented extreme binge drinking. The NS and HATM classes exhibited a higher density of relatives with a history of alcohol abuse than the other classes. Most students were classified into high-risk consumption clas- ses, characterized by co-use of substances or by heavy alcohol use. These patterns of substance use have been associated with a variety of negative consequences. Keywords: Alcohol; tobacco; marijuana; classes of substance use; college students.

\section{Resumo}

O consumo de substâncias é altamente prevalente em estudantes universitários na Argentina e no mundo. Diferenciar usuários com diferentes padrões de consumo de substâncias é importante para identificar aqueles mais vulneráveis à transição para um consumo patológico. Para isso, o objetivo foi identificar, por meio da Análise de Classe Latente aplicada a diferentes indicadores de consumo de álcool, tabaco e maconha, as classes de consumidores, e observar se essas classes diferiam na densidade de parentes com consumo abusivo de álcool e outros indicadores de consumo. A análise foi realizada com alunos do primeiro ano $(n=$ 4.497) que completaram os instrumentos para medir: 1-consumo de álcool, 2-consequências negativas do consumo de álcool e 3-histórico familiar de abuso de álcool. Foram identificadas quatro classes de usuários: 1-CATM: alto consumo de álcool, consumo de tabaco e maconha; 2-CMEA: consumo moderado / alto de álcool, sem consumo de outras substâncias; 3-CMBA: consumo moderado / baixo de álcool, sem consumo de outras substâncias; e 4-SC: nenhum uso de substância. Os membros das classes CATM e CMEA relataram níveis significativamente mais elevados de consumo de álcool do que o resto das classes. Os membros do CATM apresentaram consumo extremo de álcool. As classes $S C$ e $C A T M$ apresentaram maior densidade de familiares com abuso de álcool do que as demais classes. A maioria dos entrevistados foi classificada em categorias de consumo de alto risco, caracterizadas pelo uso concomitante de substâncias ou pelo alto consumo de álcool. Esses padrões de consumo têm sido associados a inúmeras consequências negativas.

Palavras-chave: álcool; tabaco; maconha; classes de consumo; estudantes universitários. 
El consumo de sustancias es una problemática de relevancia sanitaria tanto en Argentina (Sedronar, 2017) como en el resto del mundo (UNODC, 2016). En el ámbito internacional, el alcohol es la sustancia psicoactiva más utilizada (OMS, 2018), su uso resulta en las mayores tasas de dependencia o abuso como sustancia primaria, y la tasa más alta de admisiones a tratamiento por trastornos relacionados al consumo de sustancias (SAMHSA, 2015). En Argentina (Sedronar, 2017) y muchos otros países (ESPAD Group, 2016; Johnston et al., 2017; SAMHSA, 2015), el consumo de alcohol en los jóvenes es altamente prevalente. Algunos grupos etarios, por ejemplo, los adultos emergentes que transitan la educación universitaria (Brett et al., 2016; Pilatti et al., 2017; Van Damme et al., 2016), exhiben con mucha frecuencia un patrón de consumo excesivo denominado consumo binge.

El consumo binge se caracteriza por la ingesta de una cantidad elevada de alcohol (i.e., $>56 / 72$ gramos de alcohol para mujeres y hombres, respectivamente) en un corto periodo de tiempo (i.e., 2 horas o menos) (NIAAA, 2004). Este patrón de consumo está asociado a una mayor probabilidad de sufrir problemas académicos, desmayos, relaciones sexuales riesgosas, agresiones físicas y sexuales, conducta violenta y accidentes de tráfico (Ferreira et al., 2014; Hingson, 2010; Pilatti et al., 2016a; White \& Hingson, 2013). El consumo binge, además, incrementa la probabilidad de desarrollar trastornos por consumo de alcohol (Cservenka \& Brumback, 2017; Dawson et al., 2010), de otras sustancias (Dawson et al., 2010) y algunos problemas de salud mental como trastornos de personalidad antisocial, trastornos de ansiedad y otros trastornos afectivos (Rehm, 2011). Estudios realizados en Argentina indican que, aproximadamente el $55 \%$ de estos jóvenes ha exhibido al menos uno de estos episodios en los últimos 6 meses (Pilatti et al., 2017; Pilatti et al., 2016b).

El tabaco y la marihuana después del alcohol, son las sustancias más consumidas en Argentina (Sedronar, 2017) y el mundo (EMCDDA, 2018;
SAMHSA, 2014). Estudios locales (Pilatti et al., 2017) y de Estados Unidos (Meier et al., 2015; Schulenberg et al., 2019; Skidmore et al., 2016; Suerken et al., 2014) indican que, aproximadamente, el $50 \%$ de los estudiantes universitarios consumió tabaco alguna vez en su vida, el $30 \%$ en el último año y el $20 \%$ lo hizo durante el último mes. Por otro lado, el $7.8 \%$ de la población entre 12 y 65 años de Argentina declaró haber consumido marihuana en el último año (Sedronar, 2017). En una investigación con estudiantes universitarios (Pilatti et al., 2017) se observaron valores sensiblemente mayores a los de la población general. Específicamente, se encontró que el 36\% de los estudiantes universitarios encuestados había consumido marihuana alguna vez en su vida, mientras que el $27.5 \%$ lo había hecho durante el último año y el $17.5 \%$ en el último mes, porcentajes que fueron mayores en hombres que en mujeres. Valores similares se han observado en Estados Unidos. Por ejemplo, Suerken et al. (2014) reportaron que, para el comienzo de la universidad, el 29.5\% ya había consumido marihuana alguna vez en su vida y el $8.5 \%$ comenzó a consumirla durante el primer año de estudios universitarios.

En general, se ha encontrado que el consumo de alcohol aumenta durante la adolescencia alcanzando su pico entre los 18 y los 25 años (Chen \& Jacobson, 2013; Hagger-Johnson et al., 2013; Patrick et al., 2016; White et al., 2006), momento a partir del cual comienza a descender (Britton et al., 2015). El consumo binge y el consumo de otras sustancias como la marihuana son más frecuentes en los jóvenes que en cualquier otro grupo de edad (Farmer et al., 2015; Patrick et al., 2016; SAMHSA, 2015; Schulenberg et al., 2019; Sedronar, 2017). Dentro de esta etapa, la transición de la escuela secundaria hacia la vida universitaria ha sido señalada como un periodo crítico para el incremento en el consumo de sustancias (Cho et al., 2015; Derefinko et al., 2016; Skidmore et al., 2016). Numerosos trabajos han señalado que los estudiantes universitarios consumen significativamente más alcohol que sus pares 
que no asisten a la universidad (Chen \& Jacobson, 2013; Johnston et al., 2017; Patrick et al., 2016; Schulenberg et al., 2019) y que, comparados con la población general, los estudiantes universitarios presentan mayor riesgo de exhibir consumo binge (Krieger et al., 2018; Merrill \& Carey, 2016; Patrick, 2016). Asimismo, en una revisión reciente sobre consumo de sustancias durante la universidad se ha señalado que el consumo de marihuana aumenta durante el transcurso de los años universitarios (Skidmore et al., 2016).

Diferentes características biológicas, de personalidad, sociales y ambientales se han propuesto como factores de riesgo y protección para el consumo de alcohol. Entre ellas, un inicio temprano al consumo de alcohol o una historia familiar (HF) de abuso o dependencia al alcohol, están asociados a mayor prevalencia de consumo elevado de alcohol y de problemas causados por el alcohol u otras sustancias (Chassin et al., 2014; Khoddam et al., 2015; Pilatti et al., 2014; Pilatti et al., 2017). El inicio temprano en el consumo de alcohol usualmente se sitúa en los $\leq 14-15$ años (Pilatti et al., 2014, 2017). Este inicio temprano se asocia en diferentes muestras y culturas, a un mayor consumo de alcohol y de otras sustancias como tabaco y marihuana, y a una mayor experimentación de problemas derivados (Jackson et al., 2015; Moss et al., 2014; Pilatti et al., 2017; Sartor et al., 2016).

La operacionalización de HF, en tanto, ha variado desde considerar solo al padre o la madre biológicos (Acheson et al., 2018; Eiler et al., 2018), a cualquier familiar de primer grado (i.e., madre o padre), segundo (i.e., abuelos o hermanos) o tercero (i.e., tíos) (Brkic et al., 2015; Caneto et al., 2018). Otros trabajos estiman la densidad de familiares con abuso de alcohol (i.e., la sumatoria) como un indicador del grado de riesgo (Cservenka \& Nagel, 2012; Kendler et al., 2015; Khoddam et al., 2015). La relación entre una HF de abuso de alcohol con una mayor prevalencia de consumo elevado de alcohol y de problemas asociados al consumo de alcohol y de otras sustancias, ha sido ampliamente documentada con resultados consistentes en muestras de adolescentes (Khoddam et al., 2015), de estudiantes universitarios (Kendler et al., 2015) y de jóvenes y adultos (Chassin et al., 2004; Söderpalm Gordh et al., 2011).

La detección de individuos que exhiben patrones de consumo de alcohol elevado como el consumo binge, o que poseen factores de riesgo como HF, es importante para diferenciar aquellos que pueden progresar de un consumo social y controlado, a un consumo patológico de sustancias. Esto permitirá diseñar estrategias preventivas y abordajes terapéuticos específicos. Sin embargo, se debe progresar hacia la tipificación de grupos de consumidores conformados por múltiples indicadores de consumo y de diferentes sustancias (Gilreath et al., 2014; Göbel et al., 2016). En este contexto, el presente trabajo identificó, mediante Análisis de Clases Latentes (ACL) y en una amplia muestra de universitarios argentinos, clases de consumidores de sustancias a partir de indicadores de consumo de alcohol, tabaco y marihuana. Estas clases fueron analizadas por diferencias en variables sociodemográficas, en la densidad de familiares con abuso de alcohol, en las consecuencias negativas derivadas del consumo de alcohol, la frecuencia de intoxicación y en variables factibles de dar lugar a intervenciones, como la edad de inicio en el consumo de alcohol.

\section{Método}

\section{Participantes}

El presente estudio empleó datos recolectados en la encuesta basal (recopilados entre febrero y mayo del 2014) de la cohorte 2014 del estudio Estudio Longitudinal Sobre Alcohol (ELSA). ELSA mide el consumo de sustancias en estudiantes universitarios argentinos a lo largo de los primeros años de la universidad. 13 unidades académicas de la Universidad Nacional de Córdoba (Argentina) 
fueron contactadas, y 10 brindaron su aprobación para invitar a los estudiantes y recolectar datos en las aulas. En total, 4.497 (57.8\% mujeres, $42.2 \%$ hombres) estudiantes de primer año, con edades comprendidas entre 18 y 25 años $\left(\mathrm{M}_{\text {edad }}=19.06\right.$, $\mathrm{SD}=1.64)$, completaron la encuesta.

\section{Procedimiento}

En las aulas se invitó a los estudiantes a participar en el estudio, explicándoles que el objetivo era mejorar la comprensión de las conductas de consumo de sustancias durante los años universitarios. Los estudiantes recibieron instrucciones de no proporcionar información de identificación personal. Se enfatizó que la participación era completamente voluntaria y que los datos proporcionados serían confidenciales.

La recolección de datos se realizó aproximadamente en 35 minutos, y de manera colectiva en las aulas de dictado de clases. Los participantes dieron su consentimiento informado por escrito antes de la administración de la encuesta en formato lápiz y papel. Se instruyó a los participantes sobre cómo completar los instrumentos y procedieron a responder las preguntas. Los participantes no fueron recompensados (monetariamente o de otra manera) ni recibieron créditos académicos por su participación.

\section{Instrumentos}

Cuestionario sociodemográfico: un cuestionario ad hoc recolectó información en referencia al sexo, edad, lugar donde finalizaron sus estudios secundarios, situación laboral, horas de cursado y cantidad de hijos.

Consumo de alcohol: se empleó una versión adaptada del cuestionario ad hoc de Pilatti et al. (2021). El consumo de alcohol fue definido como el consumo de al menos un vaso de cualquier bebida alcohólica. Se indagó sobre: (i) el tipo de bebida alcohólica consumida habitualmente (e.g., cerveza, vino, vodka, ron, etcétera); (ii) la frecuencia usual de consumo durante el último año (desde menos de una vez al mes, hasta cuatro veces o más por semana) y (iii) la cantidad de vasos consumidos en una ocasión típica de consumo (desde un vaso, hasta 14 vasos o más). Quienes nunca consumieron bebidas con alcohol o que no lo hicieron durante el año anterior tenían una opción para indicarlo. En función de los contenidos alcohólicos conocidos en cada bebida y las respuestas a las preguntas uno y tres, se calculó la cantidad de gramos de alcohol consumidos por ocasión de consumo (i.e., cantidad por ocasión) que, a su vez, permitieron calcular la cantidad de unidades estándar de bebidas con alcohol consumidas por ocasión de consumo. Cada unidad estándar se consideró como equivalente a 14 gramos de alcohol (NIAAA, 2004). De igual forma, se indagó sobre la ocurrencia (en el último año y en el último mes) del consumo hasta la ebriedad, y la cantidad de episodios de consumo hasta la ebriedad durante el último mes. Una pregunta determinó la presencia de consumo binge (i.e., la ingesta de $>4 / 5$ unidades estándar de alcohol para mujeres y hombres, respectivamente, en $\leq 2$ horas) a partir de la definición del NIAAA (2004).

Edad de inicio en el consumo de alcohol: siguiendo el procedimiento descrito en Pilatti et al. (2014), se incluyó una pregunta que indagaba sobre la edad en la que consumió por primera vez un vaso o más de una bebida con alcohol.

Consumo de tabaco y marihuana: se creó un cuestionario ad hoc que evaluó (i) la ocurrencia (vida, último año/mes/semana) del consumo de tabaco, (ii) la frecuencia de consumo de tabaco (desde menos de una vez al mes, hasta cuatro veces o más por semana) y (iii) la ocurrencia de consumo de marihuana durante el último año.

Consecuencias negativas del consumo de alcohol: se utilizó la versión adaptada al español ([ s-YAACQ], Pilatti et al., 2016a) del Young Adult Alcohol Consequences Questionnaire ([YAACQ], Read et al., 2006). Este cuestionario de 48 ítems dicotómicos mide las consecuencias del consumo 
de alcohol en ocho dominios: social/interpersonal, autocontrol disminuido, autopercepción, cuidado personal, conductas riesgosas, académico/ ocupacional, dependencia fisiológica y pérdida de conocimiento. Los participantes debían indicar si, en el último año, habían experimentado las consecuencias enumeradas en los ítems. Los ítems de cada dominio se suman para obtener un puntaje en ese dominio en particular. Asimismo, puede emplearse la sumatoria de los puntajes en cada dominio, como un indicador de la cantidad de consecuencias negativas experimentadas en ese periodo. En el estudio de adaptación al medio local (Pilatti, 2016a), las puntuaciones del s-YAACQ han mostrado adecuados valores de confiabilidad (entre .70 y .91 para las subescalas), estimados a partir de correlaciones tetracóricas (Ledesma et al., 2011).

Historia familiar de abuso de alcohol: siguiendo a LaBrie et al. (2010), se preguntó si algún familiar biológico de primer grado (madre o padre), segundo (hermanos o abuelos) o tercero (tíos) tenía o había tenido una historia de abuso de alcohol que derivó, o podría haber derivado, en tratamiento. Luego, y siguiendo trabajos previos (Cservenka \& Nagel, 2012; Kendler et al., 2015; Khoddam et al., 2015) se estimó la densidad de familiares con una historia de abuso de alcohol (i.e., la sumatoria de la cantidad de familiares biológicos —en general de primer, segundo y tercer grado--).

\section{Análisis de datos}

Para identificar las clases de consumidores de sustancias, se realizó un ACL con los siguientes indicadores: (i) cantidad de unidades estándar de bebidas con alcohol consumidas por ocasión de consumo (CANT: $0=0$ unidades, $1=$ entre 1 y 2 unidades, $2=$ entre 3 y 5 unidades, $3=$ entre 6 y 10 unidades, y $4=11$ unidades o más); (ii) frecuencia de consumo usual (FREC: $0=$ sin consumo, $1=$ menos de una vez al mes, $2=$ entre una y tres veces al mes, $3=$ una vez a la semana y $4=$ dos veces a la semana o más); (iii) consumo binge (BG: $0=\sin$ consumo binge y $1=$ con consumo binge); (iv) consumo de tabaco (TAB: $0=$ sin consumo de tabaco, $1=$ entre menos de una vez al mes y tres veces a la semana y $2=$ cutaro veces por la semana o más), y (v) consumo de marihuana (MAR: $0=$ sin consumo de marihuana y $1=$ con consumo de marihuana en el último año).

El ACL identifica grupos homogéneos a su interior y heterogéneos respecto al resto, con los indicadores provistos (Percy \& Iwaniec, 2007). Se probaron modelos desde una a seis clases hasta obtener el modelo más parsimonioso. El número de clases latentes se determinó mediante la prueba Lo-Mendell-Rubin (LMR), que compara si una solución de $k$ clases presenta mejor ajuste que una solución de $k-1$ clases. A su vez, se examinaron los índices de entropía y los modelos con n-clases se compararon en función de tres medidas de bondad de ajuste y parsimonia: 1-indicador de información bayesiana (BIC), 2-indicador de información de Akaike (AIC) y 3-indicador de información de Akaike 3 (AIC3). En todos los casos, un menor valor sugiere mejor ajuste. Se analizó, además, en qué medida las clases representaban categorías diferentes (Muthén, 2006). El modelo de ACL estima dos tipos de parámetros: (i) probabilidad de pertenencia a cada clase (i.e., tamaño relativo de cada clase), y (ii) la probabilidad de respuesta de los distintos indicadores en cada una de las clases resultantes (Uebersax, 1994).

Después de decidir sobre el número apropiado de clases, se examinó dentro de cada una mediante estadística descriptiva (i.e., porcentajes, media y desviación estándar según correspondiera), las características sociodemográficas. Mediante Análisis de Varianza (ANOVA) y pruebas de Chi cuadrado se analizaron diferencias y asociaciones entre las características sociodemográficas y las clases identificadas. Asimismo, y para una mejor descripción de las clases, se llevaron a cabo ANOVA, seguidos de análisis post hoc de Tukey, para comparar a las clases de consumidores en una serie de indicadores 
de consumo de alcohol (edad de inicio en el consumo de alcohol, ocurrencia de episodios de ebriedad en el último año, y mes y frecuencia de episodios de ebriedad en el último mes), y en las consecuencias negativas derivadas del consumo de alcohol y pruebas Chi cuadrado para examinar la asociación entre la ocurrencia de episodios de intoxicación y las clases identificadas. La prueba de KruskalWallis examinó si las clases diferían en la densidad de familiares con una historia de abuso de alcohol. Esta variable exhibió valores de asimetría y kurtosis superiores a \pm 2 .

Hubo $160(3.6 \%)$ casos perdidos en la variable ocurrencia de consumo binge, por lo que la estima- ción de las clases y los análisis que las involucran se realizaron con una submuestra de $n=4.337$. El nivel $\alpha$ fue $0.05 \mathrm{y}$ los análisis se realizaron con Latent Gold 4.5 o SPSS 23.

\section{Resultados}

\section{Características sociodemográficas}

La Tabla 1 presenta las características sociodemográficas para la muestra total. Además, la información se muestra diferenciada por sexo. La mayoría $(74.8 \%)$ de los estudiantes universitarios

Tabla 1.

Características sociodemográficas para la muestra total, diferenciadas por sexo

\begin{tabular}{|c|c|c|c|}
\hline Característica & Total $(n=4.497)$ & Mujeres $(n=2.600)$ & Hombres $(\mathrm{n}=1.897)$ \\
\hline \multicolumn{4}{|l|}{ Edad } \\
\hline Edad promedio & $19.06 \pm 1.64$ & $18.95 \pm 1.58$ & $19.2 \pm 1.71$ \\
\hline 18-19 años & 74.8 & 78.1 & 70.5 \\
\hline 20-21 años & 15.1 & 13.1 & 17.8 \\
\hline 22-23 años & 6.5 & 5.6 & 7.6 \\
\hline 24-25 años & 3.6 & 3.2 & 4 \\
\hline \multicolumn{4}{|l|}{ Lugar de origen } \\
\hline Pcia. de Córdoba & 68.3 & 69.6 & 66.5 \\
\hline Otra provincia & 29.8 & 28.7 & 31.3 \\
\hline Otro país & 1.9 & 1.7 & 2.2 \\
\hline \multicolumn{4}{|l|}{ Horas de cursado } \\
\hline Menos de 10 horas & 31.2 & 34.7 & 26.2 \\
\hline Entre 11 y 20 horas & 65 & 61.1 & 70.4 \\
\hline Más de 20 horas & 3.8 & 4.2 & 3.3 \\
\hline \multicolumn{4}{|l|}{ Situación laboral } \\
\hline No trabaja & 82.6 & 85.4 & 78.7 \\
\hline Trabaja hasta 10 horas & 6.7 & 6 & 7.6 \\
\hline Trabaja más de 10 horas & 10.7 & 8.6 & 13.7 \\
\hline \multicolumn{4}{|l|}{ Hijos } \\
\hline No tiene & 95.2 & 94 & 96.9 \\
\hline Sí tiene & 4.8 & 6 & 3.1 \\
\hline
\end{tabular}

Nota: todos los valores corresponden al porcentaje de participantes ubicados en cada categoría. Para variables continuas, los datos se presentan como medias y desviación estándar de la media. 
tenía entre 18 y 19 años y eran de la provincia de Córdoba (68.3\%). Así mismo, la mayoría cursaba entre 11 y 20 horas semanales (65\%), no trabajaba $(82.6 \%)$ y no tenía hijos $(95.2 \%)$.

\section{Identificación de las clases de consumidores}

El ACL reveló que la solución de cuatro clases era la más parsimoniosa y con mejor ajuste a los datos, con clases de consumidores distintas e interpretables y con tamaños muestrales adecuados. Como puede observarse en la tabla 2, los valores de BIC, AIC y AIC3 disminuyen al crecer el número de clases. Sin embargo, el valor de entropía, el índice de ajuste LMR y la relevancia teórica de las clases, favorecieron al modelo de cuatro clases.

Una vez seleccionado el modelo de cuatro clases, se evaluó el tamaño de cada una y la probabilidad de respuesta del indicador de consumo dentro de cada una de las clases resultantes (véase figura 1 y tabla suplementaria 1). La primera clase, denominada Consumo Elevado de Alcohol con Consumo de Tabaco y Marihuana (CATM), representada con un patrón de círculos en la figura 1, estuvo conformada por el $28.5 \%$ de la muestra $(n=1.234)$ y se caracterizó por una alta probabilidad (.75) de consumir seis unidades estándar o más de alcohol por ocasión de consumo, una alta probabilidad (.69) de consumir bebidas con alcohol al menos una vez a la semana, una alta probabilidad (.86) de presentar consumo binge y una probabilidad moderada de consumir marihuana (.49) y tabaco (.34).

La segunda clase, Consumo Moderado/Elevado de Alcohol (CMEA), representada con un patrón de cruces en la figura 1, agrupó al $40 \%$ de la muestra $(\mathrm{n}=1.733)$ y se caracterizó por una probabilidad moderada (.48) de consumir seis unidades o más de alcohol por ocasión de consumo, un alta probabilidad (.81) de consumir bebidas con alcohol entre una y cuatro veces al mes, una alta probabilidad (.89) de presentar consumo binge y una alta probabilidad de no consumir tabaco (.84) o marihuana (.94).

La tercera clase, Consumo Moderado/Bajo de Alcohol (CMBA), representada con un patrón de triángulos en figura 1, agrupó al $22.2 \%$ de la muestra $(\mathrm{n}=961)$ y se caracterizó por un alta probabilidad (.63) de consumir entre una y dos unidades de alcohol por ocasión de consumo, una alta probabilidad (.93) de consumir bebidas con alcohol menos de una vez a la semana (hasta tres veces al mes), y una alta probabilidad de no haber tenido consumo binge (.92) ni consumir tabaco (.87) o marihuana (.96). Finalmente, la cuarta clase, Sin Consumo (SC), 9.4\% de la muestra $(\mathrm{n}=409)$ tenía una alta probabilidad (superior a .96) de no presentar consumo actual de sustancias. En la figura 1, esta clase se representa mediante el patrón de cuadrados.

Tabla 2.

Indicadores de bondad de ajuste para los diferentes modelos

\begin{tabular}{lcccccc}
\hline & BIC & AIC & AIC3 & Entropía & LRT & $\begin{array}{c}\text { Error } \\
\text { clasificación }\end{array}$ \\
\hline 1 clase & 42719.00 & 42642.51 & 42654.51 & 1 & .000 & .000 \\
\hline 2 clases & 38055.29 & 37940.54 & 37958.54 & .865 & .000 & .034 \\
\hline 3 clases & 36326.48 & 36173.49 & 36197.49 & .845 & .000 & .000 \\
\hline 4 clases & 35941.09 & 35749.84 & 35779.84 & .717 & .062 & .152 \\
\hline 5 clases & 35822.9 & 35653.41 & 35689.41 & .681 & .064 & .184 \\
\hline 6 clases & 35880.12 & 35612.37 & 35654.37 & .647 & .690 & .232 \\
\hline
\end{tabular}

Nota: BIC: Indicador de Información Bayesiana; AIC: Indicador de Información de Akaike; LRT: Prueba Lo-Mendell-Rubin. El modelo seleccionado se indica en negrita. 


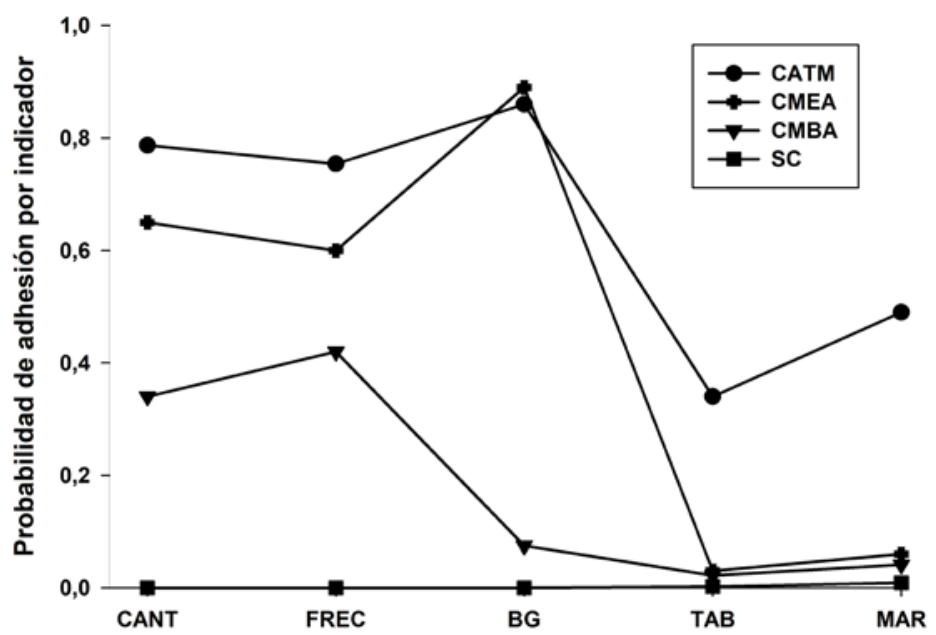

Figura 1. Probabilidad de respuesta de cada indicador de consumo dentro de cada una de las clases resultantes

Nota: clases de consumo de sustancias: CATM: Consumo Elevado de Alcohol con Consumo de Tabaco y Marihuana, CMEA: Consumo Moderado/Elevado de Alcohol, CMBA: Consumo Moderado/Bajo de Alcohol, sc: Sin Consumo. CANT: Cantidad de unidades estándar de bebidas con alcohol consumidas por ocasión de consumo, FREC: Frecuencia de consumo usual, BG: Ocurrencia de consumo binge, TAB: Frecuencia de consumo de tabaco, MAR: Ocurrencia de consumo de marihuana en el último año.

\section{Características sociodemográficas en función de las clases de consumidores}

Las clases CATM y CMEA tenían un 53.6 y $46.5 \%$ de hombres y sus miembros tenían en promedio $19.3 \pm 1.7$ y $18.8 \pm 1.4$ años, respectivamente. La clase $C M B A$ quedó conformada en su mayoría por mujeres $(73 \%)$ y sus miembros tenían $19.2 \pm 1.8$ años. Finalmente, un $68 \%$ de los miembros de la clase $S C$ eran mujeres, y los miembros de esta clase tenían 19.1 \pm 1.7 años. En las diferentes clases de consumo identificadas, la mayoría de los estudiantes (entre un 64-74\%) eran de la provincia de Córdoba.

Se encontraron asociaciones significativas entre la pertenencia a una clase y el sexo $\left(\chi^{2}=187.19\right.$, $p \leq .001)$. La inspección de la tabla de contingencia sugiere que los hombres comparados con las mujeres, tienen una menor probabilidad de pertenecer a las clases $C M B A$ y $S C$. Asimismo, se observaron diferencias significativas en la edad promedio de los miembros de las diferentes clases $(F=28.42$, $p \leq .001$ ). Los análisis post hoc mostraron que los miembros de la clase CMEA son significativamente más jóvenes que los de las otras clases.

\section{Indicadores de consumo de alcohol en función de las clases de consumidores}

La tabla 3 resume los indicadores de consumo de alcohol para las distintas clases y los análisis realizados para examinar diferencias entre dichos indicadores. Estos análisis fueron realizados -a excepción de edad de inicio en el consumo- excluyendo a la clase $S C$.

La edad de inicio en el consumo - analizada solo en aquellos participantes que habían reportado uso de alcohol en su vida - fue afectada significativamente por la clase, y los análisis post $h o c$ revelaron que todas las clases difirieron entre sí, mostrando el siguiente patrón de diferencias: $C A T M<C M E A<C M B A<S C$. Específicamente, los miembros de la clase $C A T M$ comenzaron a beber alcohol a una edad significativamente más temprana que los miembros de las otras clases, y los miembros de la clase $C M E A$ más tempranamente que los estudiantes pertenecientes a las clases $C M B A$ y $S C$. Respecto a los indicadores de consumo de alcohol, los análisis post hoc revelaron que, las tres clases con consumo de sustancias difirieron 
entre sí. Más específicamente, se encontró que los miembros de la clase CATM tuvieron más episodios de ebriedad en el último mes y experimentaron más consecuencias negativas derivadas del consumo (en todas las sub-escalas y en el total de consecuencias) que los miembros de las otras clases; y que los miembros de la clase $C M B A$ tuvieron menos episodios de ebriedad en el último mes y experimentaron menos consecuencias negativas derivadas del consumo - en todas las sub-escalas y en el total de consecuencias- que los miembros de las otras dos clases.

Asimismo, se observaron asociaciones significativas entre las clases y la ocurrencia de episodios de intoxicación en el último año y en el último mes. La exploración de la tabla de contingencia sugiere que los miembros de la clase CATM tienen una mayor probabilidad de haber experimentado episodios de intoxicación durante el último año y mes, que los miembros de las otras clases; y los miembros de la clase $C M B A$ son los que presentan las probabilidades más bajas de exhibir episodios de intoxicación.

\section{Historia familiar de abuso de alcohol en las clases de consumidores}

Se encontraron diferencias significativas entre las clases de consumidores en la densidad de familiares con abuso de alcohol $(K=29.01, p \leq .001)$. Los análisis a posteriori mostraron que los miembros de la clase $S C$ y de la clase $C A T M$, que no mostraron diferencias significativas entre sí, reportaron una mayor densidad de familiares con abuso de alcohol que los miembros de las clases CMBA y de la clase $C M E A$.

Tabla 3.

Indicadores de consumo de alcohol y de historia familiar de abuso de alcohol para las clases de consumo identificadas

\begin{tabular}{|c|c|c|c|c|c|}
\hline Indicadores & $\begin{array}{c}\text { CATM } \\
(\mathrm{n}=1.234)\end{array}$ & $\begin{array}{c}\text { CMEA } \\
(\mathrm{n}=1.733)\end{array}$ & CMBA $(n=961)$ & $\begin{array}{c}\mathrm{SC} \\
(\mathrm{n}=409)\end{array}$ & $F / K / \chi^{2}$ \\
\hline Edad de inicio consumo & $14.23 \pm 1.48$ & $14.9 \pm 1.43$ & $15.69 \pm 1.6$ & $16.01 \pm 1.61$ & $211.91^{* * *}$ \\
\hline \multicolumn{6}{|l|}{ Intoxicación } \\
\hline Ocurrió último año (\%) & 73.7 & 53.7 & 18.5 & --- & $665.01^{* * *}$ \\
\hline Ocurrió último mes (\%) & 40.6 & 18.4 & 3 & --- & $468.95^{* * *}$ \\
\hline Frecuencia último mes & $.77 \pm 1.4$ & $.28 \pm .8$ & $.04 \pm .25$ & --- & $175.37^{* * *}$ \\
\hline \multicolumn{6}{|c|}{ Consecuencias negativas último año } \\
\hline Social/Interpersonal & $2.01 \pm 1.56$ & $1.59 \pm 1.5$ & $.59 \pm .96$ & 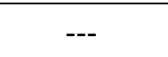 & $279.65^{* * *}$ \\
\hline Autocontrol disminuido & $2.21 \pm 1.52$ & $1.7 \pm 1.32$ & $.7 \pm .96$ & $\begin{array}{ll}-- \\
--\end{array}$ & $358.76^{* * *}$ \\
\hline Autopercepción & $.79 \pm 1.14$ & $.85 \pm 1.2$ & $.51 \pm .99$ & -- & $28.02^{* * *}$ \\
\hline Cuidado personal & $2.06 \pm 1.96$ & $1.39 \pm 1.67$ & $.46 \pm 1.02$ & --- & $251.1^{* * *}$ \\
\hline Conductas riesgosas & $1.62 \pm 1.65$ & $.90 \pm 1.19$ & $.26 \pm .62$ & -- & $314.66^{* * *}$ \\
\hline Académico/Ocupacional & $.48 \pm .9$ & $.24 \pm .6$ & $.07 \pm .32$ & -- & $102.66^{* * *}$ \\
\hline Dependencia fisiológica & $.55 \pm .65$ & $.34 \pm .57$ & $.07 \pm .28$ & --- & $207.64^{* * *}$ \\
\hline Pérdida de conocimiento & $3.35 \pm 1.71$ & $2.54 \pm 1.69$ & $.97 \pm 1.27$ & --- & $597.7^{* * *}$ \\
\hline Total consecuencias & $13.07 \pm 7.26$ & $9.56 \pm 6.42$ & $3.66 \pm 4.24$ & --- & $608.32^{* * *}$ \\
\hline Densidad HF & $.36 \pm .73$ & $.25 \pm .6$ & $.28 \pm .63$ & $.41 \pm .79$ & $29.01^{* * *}$ \\
\hline
\end{tabular}

Nota: clases de consumo de sustancias: CATM: Consumo Elevado de Alcohol con Consumo de Tabaco y Marihuana, CMEA: Consumo Moderado/Elevado de Alcohol, CMBA: Consumo Moderado/Bajo de Alcohol, sC: Sin Consumo. Densidad HF: Densidad de familiares con una historia de abuso de alcohol. 


\section{Discusión}

En los últimos años se ha evidenciado un notable aumento en el consumo de alcohol, tabaco y marihuana en la población general argentina y los jóvenes en particular (Sedronar, 2017). De igual manera, se ha sugerido que la transición de la escuela secundaria a la universidad es un periodo crítico para el aumento en el consumo de sustancias y el desarrollo de patrones problemáticos de consumo (Cho et al., 2015; Derefinko et al., 2016; Skidmore et al., 2016; Suerken et al., 2014). El presente trabajo identificó en una muestra de estudiantes universitarios argentinos, cuatro clases de consumidores a partir de indicadores de consumo de alcohol, tabaco y marihuana. Una clase exhibía consumo elevado de alcohol y consumo de tabaco y marihuana; otras dos exhibían consumo moderado/alto y moderado/bajo de alcohol, pero no consumo de otras sustancias y, finalmente, se identificó una clase sin consumo de sustancias.

Fueron pocos los estudiantes que reportaron no consumir ninguna sustancia durante los últimos 12 meses (clase $S C, 9.4 \%$ de la muestra; $4 \%$ de la muestra total reportó no haber consumido nunca en su vida). Estos resultados coinciden con trabajos previos que indican que el consumo de alcohol en jóvenes es casi normativo (ESPAD Group, 2016; Johnston et al., 2017; SAMHSA, 2015; Sedronar, 2017). A su vez, cabe destacar que las clases $C A T M$ y $C M E A$, que agrupan casi al $70 \%$ de la muestra de estudiantes universitarios examinados, presentaron niveles de consumo habitual considerablemente altos (i.e., más de seis unidades estándar de alcohol por ocasión de consumo). Es decir, cada vez que estos jóvenes consumen alcohol superan el umbral de consumo episódico elevado (Courtney \& Polich, 2009), lo que incrementa el riesgo de sufrir una amplia variedad de consecuencias negativas (Ferreira et al., 2014; White \& Hingson, 2013), incluyendo el desarrollo de trastornos por consumo de alcohol (Cservenka \& Brumback, 2017) y de otras sustancias (Dawson et al., 2010).
Estos valores son incluso bastante más elevados que los reportados en población general de Estados Unidos — donde aproximadamente el 26\% reportó consumo episódico elevado-(Dawson et al., 2015) y de Argentina - donde cerca del $20 \%$ reportó consumo episódico elevado- (Sedronar, 2017). De igual forma, los valores hallados en este trabajo superan los reportados por estudiantes universitarios argentinos (Pilatti et al., 2017; Pilatti et al., 2016b) y de otros países (Evans-Polce et al., 2017; Patrick et al., 2016; Tavolacci et al., 2013; Velázquez et al., 2011). En conjunto, los resultados evidencian que nos encontramos frente a una población particularmente vulnerable.

La clase CATM llamó nuestra atención por el alarmante patrón de consumo exhibido. Los miembros de esta clase, que agrupó al $28.5 \%$ de la muestra, indicaron que consumen aproximadamente cinco días al mes y que han experimentado, en promedio, 13 consecuencias negativas derivadas de su consumo de alcohol. Más relevante es que reportaron consumir, en promedio, casi diez unidades estándar por ocasión de consumo. Recientemente se ha propuesto que los criterios tradicionales de consumo excesivo de alcohol son demasiado bajos para identificar el consumo problemático de alcohol entre estudiantes universitarios (Hingson \& White, 2013). De hecho, se ha sugerido que los jóvenes superan significativamente este criterio, al punto de quedar obsoleto (Hingson \& White, 2013; Patrick, 2016). Nuestros resultados apoyan esta última noción. En este contexto, en los últimos años, la atención se ha trasladado al denominado "consumo extremo de alcohol", que ha sido definido como la ingesta de ocho y diez unidades estándar para mujeres y hombres, respectivamente (Hingson \& White, 2013; Mundt et al., 2009; Patrick et al., 2013; Polak \& Conner, 2012). Este patrón de consumo parece caracterizar a la clase CATM de este estudio.

En general, se ha encontrado que aquellas personas que presentan consumo extremo de alcohol exhiben más déficits físicos y cognitivos (e.g., 
problemas de concentración y pobre desempeño académico), que aquellos que exhiben consumo elevado de alcohol (Polak \& Conner, 2012). También se ha visto que el riesgo de lesiones relacionadas con el alcohol aumenta notablemente a medida que incrementan los días de consumo extremo de alcohol (i.e., más de ocho unidades estándar por ocasión de consumo), y que aquellos con consumo extremo de $\geq 4$ días por mes (e.g., cada fin de semana) tienen cinco veces más probabilidades de sufrir lesiones que aquellos que no lo hacen (Mundt et al., 2009). En esa línea, en este trabajo observamos que los miembros de la clase CATM tienen una ocurrencia marcadamente superior (al menos el doble) de algunas consecuencias negativas pertenecientes a la sub-escala de conductas riesgosas que los miembros de las otras clases (resultados no mostrados en la sección resultados), tales como "he manejado un auto cuando sabía que había tomado demasiado para manejar de manera segura" $(16.8,7.7$ y $1.9 \%$ para $C A T M$, CMEA y CMBA, respectivamente); "me he metido en peleas físicas luego de tomar alcohol" (15.2, 6.1 y $0.6 \%$ para $C A T M, C M E A$ y $C M B A$, respectivamente); "he dañado alguna propiedad, o he realizado algún acto de vandalismo" (17.6, 6.8 y 1.2\% para CATM, CMEA y CMBA, respectivamente); y "no me cuidé de enfermedades de transmisión sexual (ETS) o de la posibilidad de embarazo no deseado" $(10.3,5.5$ y $1.8 \%$ para $C A T M, C M E A$ y $C M B A$, respectivamente).

Respecto a la distribución de hombres y mujeres en las diferentes clases, las mujeres presentaron una mayor probabilidad de pertenecer a las clases $C M B A$ y $S C$. Este resultado es consistente con la literatura, que indica que los hombres usualmente exhiben mayores niveles de consumo de sustancias que las mujeres (Meier et al., 2015; Suerken et al., 2014). Sin embargo, es necesario destacar que las dos clases de mayor consumo (CATM y CMEA) concentraron cantidades similares de hombres y de mujeres $(53.6 \%$ y $45.5 \%$ de hombres en cada clase, respectivamente). Recien- temente, se ha señalado que la brecha en el consumo de hombres y mujeres está desapareciendo (Healey et al., 2014), lo cual genera gran preocupación, ya que, cantidades similares de alcohol son más dañinas entre las mujeres que entre los hombres (Erol \& Karpyak, 2015) y los trastornos por consumo de alcohol progresan más rápidamente en mujeres que en hombres (Ehlers et al., 2010; Nolen-Hoeksema, 2004).

Finalmente, los miembros de las clases SC y CATM reportaron una mayor densidad de familiares con abuso de alcohol, que los miembros de las otras dos clases. Tradicionalmente, se ha reportado que aquellos con HF y con una mayor densidad de familiares con abuso de alcohol, exhiben mayor consumo de alcohol y de otras sustancias, y mayor cantidad de problemas derivados (Acheson et al., 2018; Kendler et al., 2015; Khoddam et al., 2015; LaBrie et al., 2010), que aquellos sin HF o con menor densidad de familiares con problemas de abuso de alcohol. Los resultados del presente trabajo coinciden, parcialmente, ya que los miembros de la clase CATM exhibieron los mayores niveles de consumo y presentaron una mayor densidad de familiares con abuso de alcohol que los de las clases CMEA y CMBA. Un resultado inesperado fue que los miembros de la clase $S C$ reportaron una mayor densidad de familiares con abuso de alcohol que los miembros de las clases $C M E A$ y $C M B A$ y, además, no mostraron diferencias significativas con los miembros de la clase CATM. En esta línea, una revisión (Elliott et al., 2012) señaló que la historia familiar no se relaciona con la cantidad de consumo de alcohol, aunque sí con los trastornos por consumo de alcohol y con el consumo de otras sustancias, demostrando que esta relación — entre la historia familiar de abuso de alcohol y el consumo de alcohol — parece no ser tan lineal. De igual modo, se ha demostrado que algunas variables familiares como el monitoreo parental actúan como moderador del efecto de la vulnerabilidad genética hacia el desarrollo de conductas problemáticas con el uso de alcohol (Cope et al., 2017; Finan et al., 2018). Dicho esto, puede 
que la mayor densidad de familiares con abuso de alcohol entre los miembros de la clase $S C$ se deba a la moderación de algún factor social o familiar que no fue medido. Futuros estudios deberían considerar la posibilidad de analizar la relación entre historia familiar de abuso de alcohol y el consumo de sustancias, y su moderación por diferentes variables sociales y familiares.

Una limitación de este trabajo fue que los datos se basaron en el autoinforme del consumo de alcohol, tabaco y marihuana (del año 2013, recopilados entre febrero-mayo de 2014), lo cual puede llevar a estimaciones sesgadas. No obstante, el autoinforme retrospectivo del consumo de sustancias, es la metodología más empleada en la investigación sobre este tema. Asimismo, si bien se trabajó con una muestra amplia de estudiantes universitarios, la técnica de muestreo empleada no permite asegurar que los estudiantes que finalmente formaron parte de la muestra sean representativos de todos los estudiantes universitarios de la ciudad de Córdoba y de la Argentina, limitando la posibilidad de generalizar estos resultados. Por otro lado, el diseño transversal no permite establecer relaciones temporales entre las variables implicadas. Por último, no se analizó el uso simultáneo de sustancias. Al respecto, se ha señalado que el uso simultáneo de alcohol y marihuana se asocia a más consecuencias negativas, que el uso separado de cada una de estas sustancias (Brière et al., 2011).
Más allá de estas limitaciones, el aporte principal de este trabajo radica en identificar perfiles de consumo de alcohol, tabaco y marihuana dentro de una población particularmente vulnerable, los jóvenes que ingresan a la universidad y en un contexto sociocultural diferente al de la mayoría de los trabajos previos. De las cuatro clases de consumo identificadas, dos se caracterizaron por un consumo elevado de alcohol. Los resultados indican que una gran porción de los participantes presenta patrones problemáticos de consumo de alcohol (i.e., consumo episódico elevado) y que, incluso, un porcentaje relativamente alto de los estudiantes presenta consumo extremo de alcohol (patrón de consumo característico de una de las clases de consumo identificadas). Apenas recientemente el consumo extremo de alcohol está adquiriendo notoriedad en el campo de investigación de las conductas de consumo (Hua et al., 2020; Patrick et al., 2021). En función de los presentes resultados, consideramos que las medidas de consumo extremo de alcohol (8/10 unidades estándar para mujeres y hombres, respectivamente) deberían incluirse de manera rutinaria en los estudios que analizan el consumo de alcohol y, además, deberían destinarse más esfuerzos a la identificación de sus posibles causas y consecuencias a corto, mediano y largo plazo. Esto permitirá: (a) una detección más eficaz de aquellos individuos en riesgo, (b) diseñar estrategias de prevención más efectivas y (c) hallar nuevas vías de intervención en esta población de riesgo.

Tabla suplementaria 1 .

Probabilidad de respuesta de cada indicador de consumo para las clases identificadas

\begin{tabular}{lllll}
\hline \multicolumn{1}{c}{ Indicador } & CATM & CMEA & CMBA & SC \\
\hline CANT & & & & \\
\hline 0 (0 unidades) & 0.00 & 0.00 & 0.00 & 0.00 \\
\hline $1(1-2$ unidades $)$ & 0.01 & 0.04 & 0.63 & 0.00 \\
\hline $2(3-5$ unidades $)$ & 0.24 & 0.49 & 0.35 & 0.00 \\
\hline $3(6-10$ unidades $)$ & 0.34 & 0.31 & 0.01 & 0.00 \\
\hline
\end{tabular}




\begin{tabular}{|c|c|c|c|c|}
\hline Indicador & CATM & CMEA & CMBA & SC \\
\hline \multicolumn{5}{|l|}{ CANT } \\
\hline 4 ( $\geq 11$ unidades) & 0.41 & 0.17 & 0.00 & 0.00 \\
\hline \multicolumn{5}{|l|}{ FREC } \\
\hline $0(\sin$ consumo $)$ & 0.00 & 0.00 & 0.00 & 1.00 \\
\hline 1 (<1 vez al mes) & 0.02 & 0.08 & 0.38 & 0.00 \\
\hline 2 (1-3 veces al mes) & 0.29 & 0.55 & 0.56 & 0.00 \\
\hline 3 (1 vez a la semana) & 0.35 & 0.26 & 0.06 & 0.00 \\
\hline 4 ( $\geq 2$ veces a la semana) & 0.34 & 0.10 & 0.00 & 0.00 \\
\hline \multicolumn{5}{|l|}{ BG } \\
\hline 0 (sin consumo binge) & 0.13 & 0.11 & 0.92 & 1.00 \\
\hline 1 (con consumo binge) & 0.86 & 0.89 & 0.07 & 0.00 \\
\hline \multicolumn{5}{|l|}{$\mathrm{TAB}$} \\
\hline $0(\sin$ consumo $)$ & 0.38 & 0.84 & 0.87 & 0.96 \\
\hline 1 ( $\leq 3$ veces a la semana $)$ & 0.28 & 0.13 & 0.11 & 0.03 \\
\hline 2 ( $\geq 4$ veces a la semana) & 0.34 & 0.03 & 0.02 & 0.00 \\
\hline \multicolumn{5}{|l|}{ MAR } \\
\hline $0($ sin consumo $)$ & 0.51 & 0.94 & 0.96 & 1.00 \\
\hline 1 (con consumo) & 0.49 & 0.06 & 0.04 & 0.00 \\
\hline
\end{tabular}

Nota: clases de consumo de sustancias: CATM: Consumo Elevado de Alcohol con Consumo de Tabaco y Marihuana, CMEA: Consumo Moderado/Elevado de Alcohol, CMBA: Consumo Moderado/Bajo de Alcohol, sc: Sin Consumo. CANT: Cantidad de unidades estándar de bebidas con alcohol consumidas por ocasión de consumo, FREC: Frecuencia de consumo usual, BG: Ocurrencia de consumo binge, TAB: Frecuencia de consumo de tabaco, MAR: Ocurrencia de consumo de marihuana en el último año.

\section{Referencias}

Acheson, A., Vincent, A. S., Cohoon, A. J., \& Lovallo, W. R. (2018). Defining the phenotype of young adults with family histories of alcohol and other substance use disorders: Studies from the family health patterns project. Addictive Behaviors, 77, 247-254. https://doi.org/10.1016/j. addbeh.2017.10.014

Brett, E. I., Leavens, E. L., Miller, M. B., Lombardi, N., \& Leffingwell, T. R. (2016). Normative perceptions of alcohol-related consequences among college students. Addictive Behaviors, 58, 16-20. https://doi.org/10.1016/j.addbeh.2016.02.008
Briere, F. N., Fallu, J. S., Descheneaux, A., \& Janosz, M. (2011). Predictors and consequences of simultaneous alcohol and cannabis use in adolescents. Addictive Behaviors, 36(7), 785-788. https://doi.org/10.1016/j.addbeh.2011.02.012 Britton, A., Ben-Shlomo, Y., Benzeval, M., Kuh, D., \& Bell, S. (2015). Life course trajectories of alcohol consumption in the United Kingdom using longitudinal data from nine cohort studies. BMC Medicine, 13(1), 1-9. https://doi. org/10.1186/s12916-015-0273-z

Brkic, S., Söderpalm, B., \& Söderpalm Gordh, A. (2015). A family history of Type 1 alcoholism differentiates alcohol consumption in high 
cortisol responders to stress. Pharmacology Biochemistry and Behavior, 130, 59-66. https:// doi.org/10.1016/j.pbb.2014.12.008

Caneto, F., Pautassi, R. M., \& Pilatti, A. (2018). Ethanol-induced autonomic responses and risk taking increase in young adults with a positive family history of alcohol problems. Addictive Behaviors, 76(April), 174-181. https://doi. org/10.1016/j.addbeh.2017.08.008

Chassin, L., Flora, D. B., \& King, K. M. (2004). Trajectories of alcohol and drug use and dependence from adolescence to adulthood: The effects of familial alcoholism and personality. Journal of Abnormal Psychology, 113(4), 483-498. https://doi.org/10.1037/0021843X.113.4.483

Chassin, L., Haller, M., Lee, M., Handley, E., Bountress, K., \& Beltran, I. (2014). Familial factors influencing offspring substance use and dependence. In K. J. Sher (Ed.), The Oxford Handbook of Substance Use and Substance Use Disorders: Volume 1 (Vol. 1). Oxford University Press. https://doi.org/10.1093/oxford$\mathrm{hb} / 9780199381678.013 .008$

Chen, P., \& Jacobson, K. C. (2013). Longitudinal relationships between college education and patterns of heavy drinking: A comparison between Caucasians and African-Americans. Journal of Adolescent Health, 53(3), 356-362. https://doi. org/10.1016/j.jadohealth.2013.04.003

Cho, S. B., Llaneza, D. C., Adkins, A. E., Cooke, M., Kendler, K. S., Clark, S. L., \& Dick, D. M. (2015). Patterns of substance use across the first year of college and associated risk factors. Frontiers in Psychiatry, 6, 152. https://doi. org/10.3389/fpsyt.2015.00152

Cope, L. M., Munier, E. C., Trucco, E. M., Hardee, J. E., Burmeister, M., Zucker, R. A., \& Heitzeg, M. M. (2017). Effects of the serotonin transporter gene, sensitivity of response to alcohol, and parental monitoring on risk for problem alcohol use. Alcohol, 59, 7-16. https://doi.org/10.1016/j. alcohol.2016.12.001
Courtney, K. E., \& Polich, J. (2009). Binge drinking in young adults: Data, definitions, and determinants. Psychological Bulletin, 135(1), 142-156. https://doi.org/10.1037/a0014414

Cservenka, A., \& Brumback, T. (2017). The burden of binge and heavy drinking on the brain: Effects on adolescent and young adult neural structure and function. Frontiers in Psychology, 8, 1111. https://doi.org/10.3389/fpsyg.2017.01111

Cservenka, A., \& Nagel, B. J. (2012). Risky decision-making: An fMRI study of youth at high risk for alcoholism. Alcoholism: Clinical and Experimental Research, 36(4), 604-615. https:// doi.org/10.1111/j.1530-0277.2011.01650.x

Dawson, D. A., Compton, W. M., \& Grant, B. F. (2010). Frequency of 5+/4+ drinks as a screener for drug use and drug-use disorders*. Journal of Studies on Alcohol and Drugs, 71(5), 751760. https://doi.org/10.15288/jsad.2010.71.751

Dawson, D. A., Goldstein, R. B., Saha, T. D., \& Grant, B. F. (2015). Changes in alcohol consumption: United States, 2001-2002 to 2012-2013. Drug and Alcohol Dependence, 148, 56-61. https:// doi.org/10.1016/j.drugalcdep.2014.12.016

Derefinko, K. J., Charnigo, R. J., Peters, J. R., Adams, Z. W., Milich, R., \& Lynam, D. R. (2016). Substance use trajectories from early adolescence through the transition to college. Journal of Studies on Alcohol and Drugs, 77(6), 924-935. https://doi.org/10.15288/jsad.2016.77.924

Ehlers, C. L., Gizer, I. R., Vieten, C., Gilder, A., Stouffer, G. M., Lau, P., \& Wilhelmsen, K. C. (2010). Age at regular drinking, clinical course, and heritability of alcohol dependence in the San Francisco Family Study: A gender analysis. Journal of Drug Issues, 19(2), 101-110. https:// doi.org/10.1111/j.1521-0391.2009.00021.x

Eiler, W. J. A., Dzemidzic, M., Soeurt, C. M., Carron, C. R., Oberlin, B. G., Considine, R. V., Harezlak, J., \& Kareken, D. A. (2018). Family history of alcoholism and the human brain response to oral sucrose. NeuroImage: Clinical, 17, 1036-1046. https://doi.org/10.1016/j.nicl.2017.12.019 
Elliott, J. C., Carey, K. B., \& Bonafide, K. E. (2012). Does family history of alcohol problems influence college and university drinking or substance use? A meta-analytical review. Addiction, 107(10), 1774-1785. https://doi.org/10.1111/ j.1360-0443.2012.03903.x

EMCDDA. (2018). Informe europeo sobre drogas 2018: tendencias y novedades. Publications Office of the European Union.

Erol, A., \& Karpyak, V. M. (2015). Sex and gender-related differences in alcohol use and its consequences: Contemporary knowledge and future research considerations. Drug and Alcohol Dependence, 156, 1-13. https://doi. org/10.1016/j.drugalcdep.2015.08.023

ESPAD Group. (2016). ESPAD Report 2015: results from the european school survey project on alcohol and other drugs. Publications Office of the European Union. https://doi.org/10.2810/892603

Evans-Polce, R. J., Maggs, J. L., Staff, J., \& Lanza, S. T. (2017). The age-varying association of student status with excessive alcohol use: Ages 18 to 30 Years. Alcoholism: Clinical and Experimental Research, 41(2), 407-413. https:// doi.org/10.1111/acer.13294

Farmer, R. F., Kosty, D. B., Seeley, J. R., Duncan, S. C., Lynskey, M. T., Rohde, P., Klein, D. N., $\&$ Lewinsohn, P. M. (2015). Natural course of cannabis use disorders. Psychological Medicine, 45(1), 63-72. https://doi.org/10.1017/ S003329171400107X

Ferreira, J. A., Martins, J. S., Coelho, M. S., \& Kahler, C. W. (2014). Validation of Brief Young Adult Alcohol Consequences Questionnaire (B-YAACQ): Portuguese Version. Spanish Journal of Psychology, 17, 1-8. https://doi.org/10.1017/ sjp.2014.74

Finan, L. J., Simpson, E., Schulz, J., \& Ohannessian, C. M. (2018). Parental problem drinking and emerging adult problem behavior: The moderating role of parental support. Journal of Child and Family Studies, 27(4), 1175-1185. https://doi.org/10.1007/s10826-017-0953-8
Gilreath, T. D., Astor, R. A., Estrada, J. N., Johnson, R. M., Benbenishty, R., \& Unger, J. B. (2014). Substance use among adolescents in California: A latent class analysis. Substance Use \& Misuse, 49(1-2), 116-123. https://doi.org/10.3 109/10826084.2013.824468

Göbel, K., Scheithauer, H., Bräker, A. B., Jonkman, H., \& Soellner, R. (2016). Substance use patterns among adolescents in Europe: A latent class analysis. Substance Use \& Misuse, 51(9), 1130-1138. https://doi.org/10.3109/10826084.2016.1160120

Hagger-Johnson, G., Bell, S., Britton, A., Cable, N., Conner, M., O’Connor, D. B., Shickle, D., Shelton, N., \& Bewick, B. M. (2013). Cigarette smoking and alcohol drinking in a representative sample of English school pupils: Cross-sectional and longitudinal associations. Preventive Medicine, 56(5), 304-308. https:// doi.org/10.1016/j.ypmed.2013.02.004

Healey, C., Rahman, A., Faizal, M., \& Kinderman, P. (2014). Underage drinking in the UK: Changing trends, impact and interventions. A rapid evidence synthesis. International Journal of Drug Policy, 25(1), 124-132. https://doi. org/10.1016/j.drugpo.2013.07.008

Hingson, R. W. (2010). Magnitude and prevention of college drinking and related problems. Alcohol Research \& Health, 33(1-2), 45-54. https://doi. org/2010-23622-005

Hingson, R. W., \& White, A. (2013). Trends in extreme binge drinking among us high school seniors. JAMA Pediatrics, 167(11), 996-998. https:// doi.org/10.1001/jamapediatrics.2013.3083

Hua, J. P., Sher, K. J., Boness, C. L., Trela, C. J., McDowell, Y. E., Merrill, A. M., Piasecki, T. M., \& Kerns, J. G. (2020). Prospective study examining the effects of extreme drinking on brain structure in emerging adults. Alcoholism: Clinical and Experimental Research, 44(11), 2200-2211. https://doi.org/10.1111/acer.14446

Jackson, K. M., Barnett, N. P., Colby, S. M., \& Rogers, M. L. (2015). The prospective association between sipping alcohol by the sixth grade and 
later substance Use. Journal of Studies on Alcohol and Drugs, 76(2), 212-221. https://doi. org/10.15288/jsad.2015.76.212

Johnston, L. D., O’Malley, P. M., Miech, R. A., Bachman, J. G., \& Schulenberg, J. (2017). Monitoring the Future national survey results on drug use, 1975-2016: Overview, key findings on adolescent drug use. Institute for Social Research, The University of Michigan.

Kendler, K. S., Edwards, A., Myers, J., Cho, S. B., Adkins, A., \& Dick, D. (2015). The predictive power of family history measures of alcohol and drug problems and internalizing disorders in a college population. American Journal of Medical Genetics Part B: Neuropsychiatric Genetics, 168(5), 337-346. https://doi.org/10.1002/ ajmg.b. 32320

Khoddam, R., Worley, M., Browne, K. C., Doran, N., \& Brown, S. A. (2015). Family history density predicts long term substance use outcomes in an adolescent treatment sample. Drug and Alcohol Dependence, 147, 235-242. https://doi. org/10.1016/j.drugalcdep.2014.11.009

Krieger, H., Young, C. M., Anthenien, A. M., \& Neighbors, C. (2018). The epidemiology of binge drinking among college-age individuals in the United States. Alcohol Research: Current Reviews, 39(1), 23-30. https://doi.org/201905003-002

LaBrie, J. W., Migliuri, S., Kenney, S. R., \& Lac, A. (2010). Family history of alcohol abuse associated with problematic drinking among college students. Addictive Behaviors, 35(7), 721-725. https://doi.org/10.1016/j. addbeh.2010.03.009

Ledesma, R. D., Macbeth, G., \& Valero-Mora, P. (2011). Software for computing the tetrachoric correlation coefficient. Revista Latinoamericana de Psicología, 43, 181-189. https://doi. org/10.14349/rlp.v43i1.459

Meier, E. M., Tackett, A. P., Miller, M. B., Grant, D. M., \& Wagener, T. L. (2015). Which nicotine products are gateways to regular use? First-tried tobacco and current use in college students. American Journal of Preventive Medicine, 48(1), 86-93. https://doi.org/10.1016/j.amepre.2014.09.018

Merrill, J. E., \& Carey, K. B. (2016). Drinking over the lifespan: Focus on college ages. Alcohol Research: Current Reviews, 38(1), 103-14. https://doi.org/2016-30780-013

Moss, H. B., Chen, C. M., \& Yi, H. (2014). Early adolescent patterns of alcohol, cigarettes and marijuana polysubstance use and young adult substance use outcomes in a nationally representative sample. Drug and Alcohol Dependence, 136(1), 51-62. https://doi.org/10.1016/j. drugalcdep.2013.12.011

Mundt, M. P., Zakletskaia, L. I., \& Fleming, M. F. (2009). Extreme college drinking and alcoholrelated injury risk. Alcoholism: Clinical and Experimental Research, 33(9), 1532-1538. https:// doi.org/10.1111/j.1530-0277.2009.00981.x

Muthén, B. (2006). Should substance use disorders be considered as categorical or dimensional? Addiction, 101, 6-16. https://doi.org/10.1111/ j.1360-0443.2006.01583.x

NIAAA. (2004). NIAAA council approves definition of binge drinking. NIAAA Newsletter, 3. http:// pubs.niaaa.nih.gov/publications/Newsletter/ winter2004/Newsletter_Number3.pdf

Nolen-Hoeksema, S. (2004). Gender differences in risk factors and consequences for alcohol use and problems. Clinical Psychology Review, 24(8), 981-1010. https://doi.org/10.1016/j. cpr.2004.08.003

OMS. (2018). Global status report on alcohol and health 2018. Organización Mundial de la Salud.

Patrick, M. E. (2016). A call for research on high-intensity alcohol use. Alcoholism: Clinical and Experimental Research, 40(2), 256-259. https:// doi.org/10.1111/acer.12945

Patrick, M. E., Schulenberg, J. E., Martz, M. E., Maggs, J. L., O’Malley, P. M., \& Johnston, L. D. (2013). Extreme binge drinking among 12th-grade students in the United States: 
Prevalence and Predictors. JAMA pediatrics, 167(11), 1019-1025. https://doi.org/10.1001/ jamapediatrics.2013.2392

Patrick, M. E., Terry-McElrath, Y. M., \& Bonar, E. E. (2021). Patterns and predictors of high-intensity drinking and implications for intervention. Psychology of Addictive Behaviors. https://doi. org/10.1037/adb0000758

Patrick, M. E., Terry-McElrath, Y. M., Kloska, D. D., \& Schulenberg, J. (2016). High-Intensity drinking among young adults in the United States: Prevalence, frequency, and developmental change. Alcoholism: Clinical and Experimental Research, 40(9), 1905- 1912. https:// doi.org/10.1111/acer.13164

Percy, A., \& Iwaniec, D. (2007). The validity of a latent class typology of adolescent drinking patterns. Irish Journal of Psychological Medicine, 24(1), 13-18. https://doi.org/10.1016/j.adolescence.2015.07.005

Pilatti, A., Caneto, F., Garimaldi, J. A., Vera, B. D. V., \& Pautassi, R. M. (2014). Contribution of time of drinking onset and family history of alcohol problems in alcohol and drug use behaviors in Argentinean college students. Alcohol and Alcoholism, 49(2), 128-137. https:// doi.org/10.1093/alcalc/agt176

Pilatti, A., Ensinck, G., Rivarola Montejano, G., \& Pautassi, R. M. (2021). Validación de mediciones retrospectivas del consumo de alcohol mediante diarios de consumo. Psykhe., 30(1), 1-13. https://doi.org/10.7764/psykhe.2019.22311

Pilatti, A., Read, J. P., \& Caneto, F. (2016). Validation of the Spanish version of the Young Adult Alcohol Consequences Questionnaire (S-YAACQ). Psychological Assessment, 28(5), e49- e61. https://doi.org/10.1037/pas0000140

Pilatti, A., Read, J. P., \& Pautassi, R. M. (2017). ELSA 2016 cohort: Alcohol, tobacco, and marijuana use and their association with age of drug use onset, risk perception, and social norms in Argentinean college freshmen. Frontiers in Psychology, 8, 1452. https://doi.org/10.3389/fpsyg.2017.01452
Pilatti, A., Rivarola Montejano, G., Lozano, O. M., \& Pautassi, R. M. (2016). Relación entre impulsividad y consumo de alcohol en hombres y mujeres argentinos. Quaderns de Psicologia, 18(1), 7591. https://doi.org/10.5565/rev/qpsicologia.1329

Polak, M. A., \& Conner, T. S. (2012). Impairments in daily functioning after heavy and extreme episodic drinking in university students. Drug and Alcohol Review, 31(6), 763-769. https://doi. org/10.1111/j.1465-3362.2012.00429.x

Read, J. P., Kahler, C. W., Strong, D. R., \& Colder, C. R. (2006). Development and preliminary validation of the young adult alcohol consequences questionnaire. Journal of Studies on Alcohol, 67(1), 169-177. https://doi.org/10.15288/ jsa.2006.67.169

Rehm, J. (2011). The risks associated with alcohol use and alcoholism. Alcohol Research \& Health, 34(2), 135-143. https://www.ncbi.nlm.nih.gov/ pmc/articles/PMC3307043/

SAMHSA. (2014). Results from the 2013 National Survey on Drug Use and Health: Summary of national findings. Substance Abuse and Mental Health Services Administration.

SAMHSA. (2015). Behavioral health trends in the United States: Results from the 2014 National Survey on Drug Use and Health. Substance Abuse and Mental Health Services Administration.

Sartor, C. E., Lynskey, M. T., Bucholz, K. K., Madden, P. A. F., Martin, N. G., \& Heath, A. C. (2009). Timing of first alcohol use and alcohol dependence: Evidence of common genetic influences. Addiction (Abingdon, England), 104(9), 1512-1518. https://doi.org/10.1111/ j.1360-0443.2009.02648.x

Schulenberg, J. E., Johnston, L. D., O’Malley, P. M., Bachman, J. G., Miech, R. A., \& Patrick, M. E. (2019). Monitoring the Future national survey results on drug use, 1975-2018: Volume II, College students and adults ages 19-60. Ann Arbor, Institute for Social Research, The University of Michigan. http://monitoringthefuture.org/pubs.html\#monographs 
Sedronar. (2017). Estudio nacional en población de 12 a 65 años sobre consumo de sustancias psicoactivas: Magnitud del consumo de sustancias a nivel nacional. Secretaría de Políticas Integrales sobre Drogas de la Nación Argentina. https://onaf.uda. edu.ar/estudio-nacional-de-Sedronar-sobre-consumo-de-sustancias-psicoactivas-con-resultados-desagregados-para-mendoza/

Skidmore, C. R., Kaufman, E. A., \& Crowell, S. E. (2016). Substance use among college students. Child and Adolescent Psychiatric Clinics of North America, 25(4), 735-753. https://doi. org/10.1016/j.chc.2016.06.004

Söderpalm Gordh, A. H. V., Brkic, S., \& Söderpalm, B. (2011). Stress and consumption of alcohol in humans with a type 1 family history of alcoholism in an experimental laboratory setting. Pharmacology Biochemistry and Behavior, 99(4), 696-703. https://doi.org/10.1016/j. pbb.2011.05.028

Suerken, C. K., Reboussin, B. A., Sutfin, E. L., Wagoner, K. G., Spangler, J., \& Wolfson, M. (2014). Prevalence of marijuana use at college entry and risk factors for initiation during freshman year. Addictive Behaviors, 39(1), 302-307. https:// doi.org/10.1016/j.addbeh.2013.10.018

Tavolacci, M. P., Ladner, J., Grigioni, S., Richard, L., Villet, H., \& Dechelotte, P. (2013). Prevalence and association of perceived stress, substance use and behavioral addictions: A cross-sectional study among university students in France, 2009-2011. BMC Public Health, 13(1), 724. https://doi.org/10.1186/1471-2458-13-724
Uebersax, J. (1994). Latent class analysis of substance use patterns. In L.M. Collins \& L.A. Seitz (Eds.), Advances in Data Analysis for Prevention Intervention Research (NIDA Research Monograph) (pp. 64-80). National Institute on Drug Abuse.

UNODC. (2016). Informe Mundial Sobre las Drogas 2016. Oficina de las Naciones Unidas contra la Droga y el Delito.

Van Damme, J., Hublet, A., De Clercq, B., McAlaney, J., Van Hal, G., Rosiers, J., Maes, L., \& Clays, E. (2016). Context matters: Student-perceived binge drinking norms at faculty-level relate to binge drinking behavior in higher education. Addictive Behaviors, 59, 89-94. https://doi. org/10.1016/j.addbeh.2016.03.011

Velázquez, C. E., Pasch, K. E., Laska, M. N., Lust, K., Story, M., \& Ehlinger, E. P. (2011). Differential prevalence of alcohol use among 2-year and 4-year college students. Addictive Behaviors, 36(12), 1353-1356. https://doi.org/10.1016/j. addbeh.2011.07.037

White, A. M., Kraus, C. L., \& Swartzwelder, H. S. (2006). Many college freshmen drink at levels far beyond the binge threshold. Alcoholism: Clinical and Experimental Research, 30(6), 1006-1010. https://doi.org/10.1111/j.15300277.2006.00122.x

White, A., \& Hingson, R. W. (2013). The burden of alcohol use: Excessive alcohol consumption and related consequences among college students. Alcohol Research: Current Reviews, 35(2), 201218. https://doi.org/10.1037/t69599-000 